\title{
Strain-specific variation in the dnaJ gene of mycobacteria
}

\author{
T. C. VICTOR, A. M. JORDAAN, E. J. VAN SCHALKWYK*, G. J. COETZEE* and P. D. VAN HELDEN \\ MRC Centre for Molecular and Cellular Biology, Department of Medical Physiology and Biochemistry, \\ University of Stellenbosch Medical School, PO Box 19063, 7505 Tygerberg and * South African Institute for \\ Medical Research, Cape Town 8000, South Africa
}

\begin{abstract}
The polymerase chain reaction-single strand conformation polymorphism (PCR-SSCP) technique was evaluated for species identification among mycobacteria by analysis of the dnaJ gene. Nine clinical isolates of Mycobacterium tuberculosis with different fingerprint patterns all gave the same distinct SSCP banding pattern and could be distinguished from other mycobacteria, such as $M$. avium. In contrast, considerable strain-specific dnaJ gene variations were observed amongst 42 clinical isolates of $M$. avium and 13 other atypical mycobacterial strains. Only $62 \%$ of the $M$. avium isolates hybridised to an $M$. avium-specific probe and only $14 \%$ could be identified correctly as $M$. avium by both probe and restriction fragment length polymorphism analysis. This finding was supported by direct sequence analysis. Variations were also observed in $M$. gordonae and $M$. scrofulaceum isolates. Computerised analysis of $M$. avium samples broadly identified three clusters. Results suggest that although the SSCP procedure may be useful for distinguishing $M$. tuberculosis from other mycobacteria, this technique applied to the dnaJ gene may not be suitable for strain identification. The results stress the importance of testing a large collection of clinical isolates before new molecular procedures are introduced into routine laboratories.
\end{abstract}

\section{Introduction}

The causative agents of leprosy and tuberculosis are amongst the best known human pathogens. However, the clinical importance of several other mycobacterial species has increased recently, particularly as a result of the spread of the human immunodeficiency virus [1]. Conventional bacteriological identification of mycobacterial species usually requires 3-8 weeks for culture and a further 2-4 weeks for biochemical analysis; thus, it is both time-consuming and labour intensive. Serotyping has been widely used to differentiate Mycobacterium avium strains, but this method has several limitations [2-5]. Molecular hybridisation methods have also been used for species identification, and the polymerase chain reaction (PCR) technology has not only enhanced the sensitivity of detection of mycobacteria but has also led to the development of

Received 8 March 1995; revised version received 12 Aug. 1995; accepted 9 Oct. 1995.

Corresponding author: Dr T.C. Victor. new strategies to detect specific sequences [6-10]. Genus-specific targets such as the $65-\mathrm{kDa}$ heat shock protein-encoding gene [9], the dnaJ heat shock protein gene $[7,8]$ and ribosomal DNA genes [10] have been used for this purpose. Results from these studies suggest that by using a combination of PCR amplification and restriction enzyme or dot-blot analysis with species-specific probes, it is possible to differentiate between reference strains of most of the clinically important mycobacterial species. The identification of the species present in a mycobacterial infection is important, as although non-tuberculous species may be less pathogenic than $M$. tuberculosis, they can cause clinically serious diseases [11]. Furthermore, the antibiotic susceptibility of some of these species may be quite different to that of $M$. tuberculosis. Therefore, there is a growing need to identify mycobacterial isolates to species level.

Single strand conformation polymorphism (SSCP) analysis is a technique that can be used to screen for variations in amplified DNA sequences in eukaryotic [12] and prokaryotic [13] systems. The PCR-SSCP technique has been investigated in this 
study, as an alternative to biochemical testing or restriction enzyme and dot-blot analysis, to facilitate the detection of amplified $d n a J$ sequences for species identification. Samples included known reference cultures and clinical mycobacterial isolates originating from a geographical area with a high incidence of tuberculosis [14].

\section{Materials and methods}

\section{Mycobacterial isolates}

M. intracellulare ATCC 13950, M. fortuitum ATCC 6841, M. scrofulaceum ATCC 19981, M. gordonae ATCC 14470, M. bovis ATCC 19210 and $M$. avium ATCC 15679 and ATCC 35717 strains were obtained

Table 1. Molecular data of Mycobacterium isolates

\begin{tabular}{|c|c|c|c|c|}
\hline Isolate no. & Species* & Probe & $H$ infl & NaeI \\
\hline 1 & M. avium & + & + & - \\
\hline 2 & M. gordonae & - & ND & ND \\
\hline 3 & M. gordonae & - & ND & ND \\
\hline 4 & M. avium & + & + & - \\
\hline 5 & M. avium & - & - & - \\
\hline 6 & M. gordonae & + & + & + \\
\hline 7 & M. avium & + & + & + \\
\hline 8 & M. avium & + & + & + \\
\hline 9 & M. avium & - & - & - \\
\hline 10 & M. avium & - & + & - \\
\hline 11 & M. avium & + & + & - \\
\hline 12 & M. avium & + & - & + \\
\hline 13 & M. avium & + & + & - \\
\hline 14 & M. avium & + & - & - \\
\hline 15 & M. avium & + & - & - \\
\hline 16 & M. avium & + & - & + \\
\hline 17 & M. avium & + & - & + \\
\hline 18 & M. avium & - & + & - \\
\hline 19 & M. avium & + & + & - \\
\hline 20 & M. avium & + & + & + \\
\hline 21 & M. avium & + & + & - \\
\hline 22 & M. avium & - & + & - \\
\hline 23 & M. gordonae & - & ND & ND \\
\hline 24 & M. avium & + & + & + \\
\hline 25 & M. avium & + & + & + \\
\hline 26 & M. gordonae & + & - & + \\
\hline 27 & M. avium & - & + & - \\
\hline 28 & M. avium & + & + & + \\
\hline 29 & M. avium & - & + & - \\
\hline 30 & M. avium & - & - & - \\
\hline 31 & M. scrofulaceum & - & ND & ND \\
\hline 32 & M. avium & _- & + & - \\
\hline 33 & M. avium & + & - & - \\
\hline 34 & M. avium & + & - & - \\
\hline 35 & M. avium & + & + & - \\
\hline 36 & M. gordonae & - & ND & ND \\
\hline 37 & M. gordonae & - & ND & ND \\
\hline 38 & M. avium & - & - & - \\
\hline 39 & M. avium & + & - & + \\
\hline 40 & M. gordonae & - & ND & ND \\
\hline 41 & M. avium & - & - & - \\
\hline 42 & M. avium & - & - & - \\
\hline 43 & M. gordonae & - & ND & ND \\
\hline 44 & M. avium & + & - & - \\
\hline 45 & M. avium & - & + & - \\
\hline 46 & M. scrofulaceum & - & ND & ND \\
\hline 47 & M. avium & + & - & - \\
\hline 48 & M. avium & + & + & - \\
\hline 49 & M. gordonae & - & ND & ND \\
\hline 50 & M. avium & - & - & - \\
\hline 51 & M. avium & + & - & + \\
\hline 52 & M. avium & + & + & - \\
\hline 53 & M. avium & - & + & - \\
\hline 54 & M. avium & - & - & - \\
\hline 55 & M. gordonae & - & ND & ND \\
\hline 56 & M. avium (ATCC 15679) & + & + & + \\
\hline 57 & M. avium (ATCC 35717) & + & + & + \\
\hline 58 & M. scrofulaceum (ATCC 19981) & - & ND & ND \\
\hline 59 & M. intracellulare (ATCC 13950) & - & ND & ND \\
\hline 60. & M. fortuitum (ATCC 6841) & - & ND & ND \\
\hline 61 & M. gordonae (ATCC 14470) & - & ND & ND \\
\hline 62 & M. bovis (ATCC 19210) & - & ND & ND \\
\hline
\end{tabular}

$\mathrm{ND}$, not done; + or - indicates hybridisation or restricted fragments detected after digestion.

*Biochemical identification. 
from the American Type Culture Collection (ATCC), Rockville, MD, USA. Atypical mycobacteria from 55 clinical samples, isolated by Bactec (Becton Dickinson) culture and subcultured on Lowenstein Jensen medium, were identified on the basis of colony morphology, pigmentation, growth rate $\left(\right.$ at $25,32,37,42$ and $45^{\circ} \mathrm{C}$ ) and biochemical profile (niacin production, nitrate reduction, tellurite reduction, Tween hydrolysis and urease production) [15]. Atypical mycobacterial isolates analysed consisted mainly of $M$. avium (42 of 55, Table 1). Nine clinical isolates typical of $M$. tuberculosis were included as controls in the SSCP assay.

Clinical isolates were received at the SAIMR laboratory in Cape Town, South Africa, for routine identification of mycobacteria. Slant cultures were incubated at $80^{\circ} \mathrm{C}$ for $45 \mathrm{~min}$ to kill the bacteria, after which DNA was extracted with chloroform:phenol as described previously [16].

\section{PCR-SSCP procedure}

Genus-specific primers, described previously [7], were used to amplify a 236-bp region of the dnaJ gene of the mycobacterial isolates. Amplification was confirmed by electrophoretic analysis of $10 \mu \mathrm{l}$ of the 236bp PCR product on polyacrylamide $12 \%$ gels. For SSCP analysis, equal volumes of 'Stop' solution (formamide 95\%, $20 \mathrm{mM}$ EDTA and $0.005 \%$ each of bromo-phenol blue and xylene-cyanol) and amplified products ( $10 \mu \mathrm{l}$ each) were mixed and heat-denatured at $95^{\circ} \mathrm{C}$ for $2 \mathrm{~min}$. The denatured products were electrophoresed with non-denaturing PAGE $6 \%$ slab-gels, containing glycerol 5\% [12]. Electrophoresis was done at a constant $50 \mathrm{~W}$ in $0.6 \times$ TBE-buffer $(10 \times \mathrm{TBE}=900 \mathrm{M}$ each of Tris and boric acid and $25 \mathrm{mM}$ EDTA) for $4-5 \mathrm{~h}$, with cooling from a benchtop fan. FMC Gelbond film was used to manipulate the gels. The gels were then fixed for $6 \mathrm{~min}$ in a buffer containing acetic acid $0.5 \%$ and ethanol $10 \%$ and then placed in a silver nitrate $0.1 \%$ buffer for $10 \mathrm{~min}$, after which staining was done on a horizontal orbital shaker for $20 \mathrm{~min}$ or until bands were visible. The staining buffer consisted of $0.1 \mathrm{M} \mathrm{NaBH}_{4}, 1.5 \mathrm{M} \mathrm{NaOH}$ and formamide $0.004 \%$. Fixing was done in a $\mathrm{Na}_{2} \mathrm{CO}_{3}$ $0.75 \%$ buffer for $10 \mathrm{~min}$. The relative mobility of the bands generated in the SSCP procedure from clinical isolates was correlated with SSCP results from ATCC reference cultures. The bands were digitised and analysed by computer with a Genius GT1212A digitising tablet and specially designed software as described previously [16].

\section{Dot-blot and restriction enzyme analysis}

An oligonucleotide specific for $M$. avium [7], was labelled at the $5^{\prime}$ end with $\mathrm{T} 4$ polynucleotide kinase and labelled and unlabelled oligonucleotide were separated with a spin column. For dot-blot analysis, $3 \mu \mathrm{l}$ of PCR product mixture of the dnaJ gene were denatured with alkaline solution, spotted on to Hybond $\mathrm{N}$ nylon membrane and hybridised with the labelled oligonucleotide as described previously [7]. For RFLP analysis, $10 \mu \mathrm{l}$ of the amplified PCR products were digested with NaeI and Hinfl in separate reactions according to the manufacturers' specifications. After digestion, the reaction mixtures were electrophoresed on polyacrylamide $12 \%$ gels and visualised by silver staining.

\section{Sequence analysis}

The identity of the amplified DNA of target sequences of the $d n a J$ gene was confirmed in some samples by nucleotide sequence analysis by the dideoxy chaintermination method according to the manufacturer's specifications (USB; Sequence PCR product sequencing kit). Samples used for sequence analysis were amplified in duplicate and sequenced with both forward and reverse primers.

\section{DNA fingerprinting analysis}

M. tuberculosis strains were all fingerprinted by IS 6110 methodology [17]. Other isolates were fingerprinted by an oligonucleotide fingerprinting method similar to that described for M. tuberculosis [16].

\section{Results}

\section{PCR-SSCP analysis for differentiation of mycobacterial species}

$M$. tuberculosis complex (M. tuberculosis and $M$. bovis) strains could be differentiated from the reference ATCC cultures of $M$. intracellulare, $M$. fortuitum, $M$. scrofulaceum, $M$. gordonae and $M$. avium by PCRSSCP analysis (Fig. 1A). Differences were observed not only between the different species used in this study, but also between the two reference cultures of $M$. avium. These differences were consistent and reproducible. A sample result for the clinical isolates (Fig. 1A) indicates that no constant pattern was obtained for any species of atypical mycobacteria. In two clinical isolates of $M$. gordonae (samples 37 and 40), the amplified region was clearly smaller (c. 3-10 bp) than the expected 236-bp product. Results for the atypical mycobacteria contrasted with a highly constant and distinct banding pattern seen in the amplified dnaJ gene fragment from clinical isolates of $M$. tuberculosis (Fig. 1B). It should be noted that although the double strand amplified products migrated close to the 234-bp molecular marker in both Figs 1A and 1B, the banding pattern is the same for $M$. tuberculosis in both these experiments. This phenomenon is possibly due to slight differences in gel conditions and is frequently seen in SSCP analysis. All the $M$. tuberculosis isolates originated from different patients and were distinguishable from each other by IS6110-generated DNA fingerprint patterns (fingerprint results not shown). 


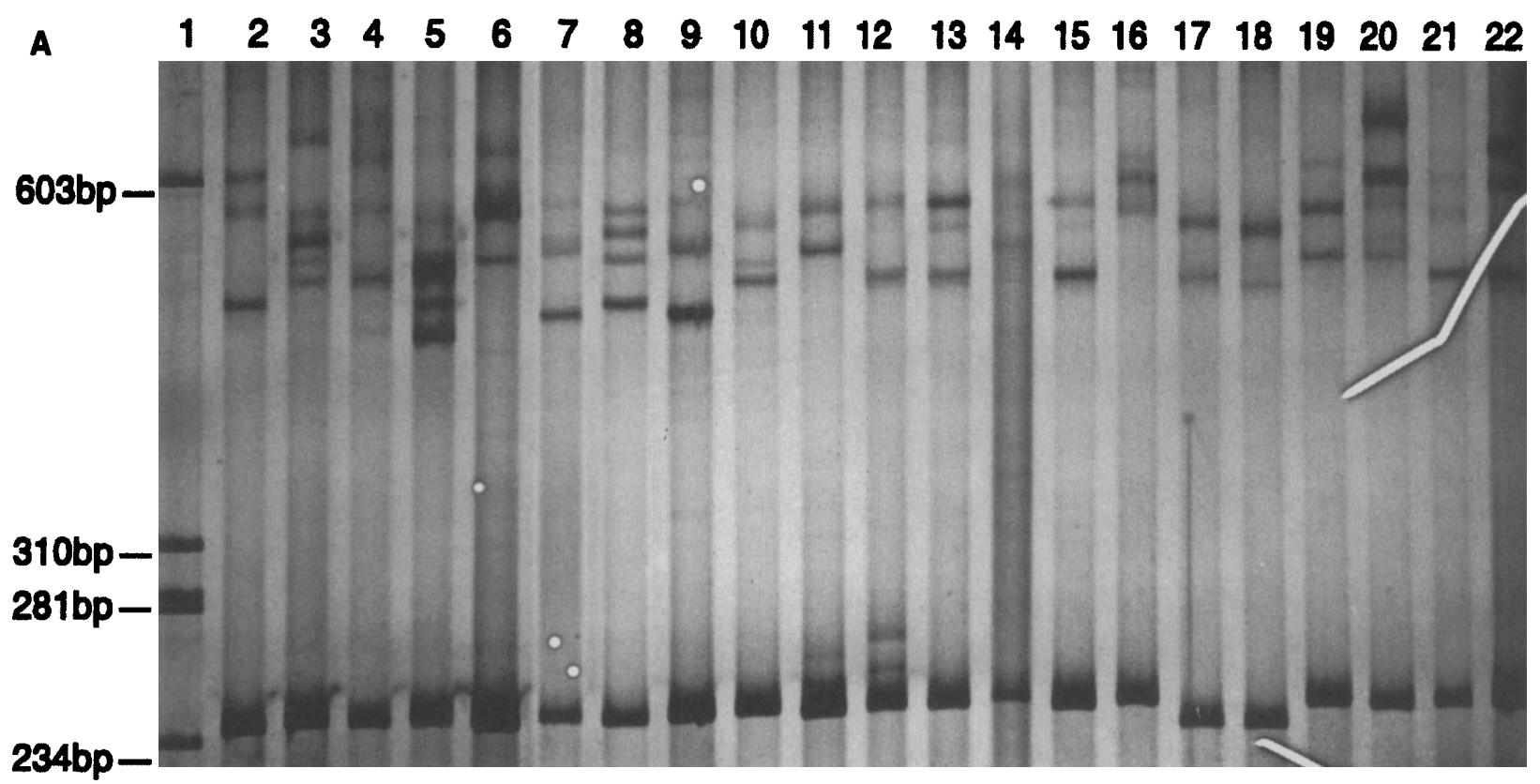

B $\begin{array}{llllllllll}1 & 2 & 3 & 4 & 5 & 6 & 7 & 8 & 9 & 10\end{array}$

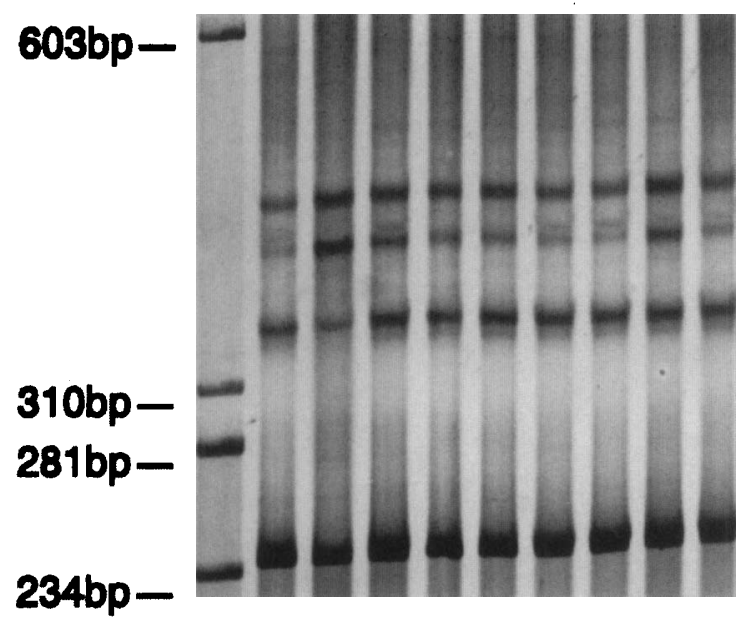

Fig. 1. PCR-SSP analysis. Reference standards and clinical isolates of mycobacteria were amplified with genusspecific primers for the dnaJ gene and the amplified products were analysed by SSCP analysis. Samples were loaded as follows: A, PhiX/Hae III (lane 1); $M$. bovis ATCC 19210 (2); $M$. intracellulare ATCC 13950 (3); $M$. fortuitum ATCC 6841 (4); M. scrofulaceum ATCC 19981 (5); $M$. gordonae ATCC 14470 (6); $M$. avium ATCC 35717 and $\operatorname{ATCC} 15679(7,8) ; M$. avium clinical isolates $7,9,10,12,14,18,39(9-15) ; M$. gordonae clinical isolates $23,37,40,55$ (16-19); $M$. scrofulaceum clinical isolates $31,46(20,21)$; clinical isolate of $M$. tuberculosis (22); B, PhiX/Hae III (lane 1) and clinical isolates of $M$. tuberculosis (2-10).

Based on the dnaJ SSCP patterns, clinical isolates of M. avium were clustered into groups A (three identical bands), B (two identical bands) and C (the remainder, unmatched, indicating two, three or more bands). Ten samples gave banding patterns characteristic of group A and 13 and 19 samples were in groups B and C, respectively. Some of the $M$. avium isolates gave results that correlated with the banding pattern of the reference $M$. avium strains (e.g., lanes 7 and 9 in Fig. 1A). However, DNA fingerprinting of 25 of 42 of the $M$. avium isolates showed that no two cultures were alike (data not shown). This and the divergent sources of the cultures make it highly unlikely that any of the strains were identical. The SSCP patterns of clinical isolates of $M$. scrofulaceum and $M$. gordonae also differed from each other. Because of the limited number of isolates of these two atypical species, no meaningful grouping could be obtained for them.

\section{Identification of clinical isolates by a probe specific for $M$. avium}

Only $26(62 \%)$ of the 42 clinical isolates of $M$. avium hybridised strongly to the $M$. avium-specific probe (Fig. 2). A positive signal was also obtained from the reference strains of $M$. avium (ATCC 15679 and 35717) and two clinical isolates identified as $M$. gordonae (nos. 6 and 26). None of the other atypical reference (ATCC) strains or clinical isolates of $M$. scrofulaceum gave a positive signal.

\section{RFLP analysis}

RFLP analysis (Fig. 3) of the amplified dnaJ gene fragment with two restriction endonucleases specific for $M$. avium in this amplified region [8] showed that, in addition to the reference cultures of $M$. avium 


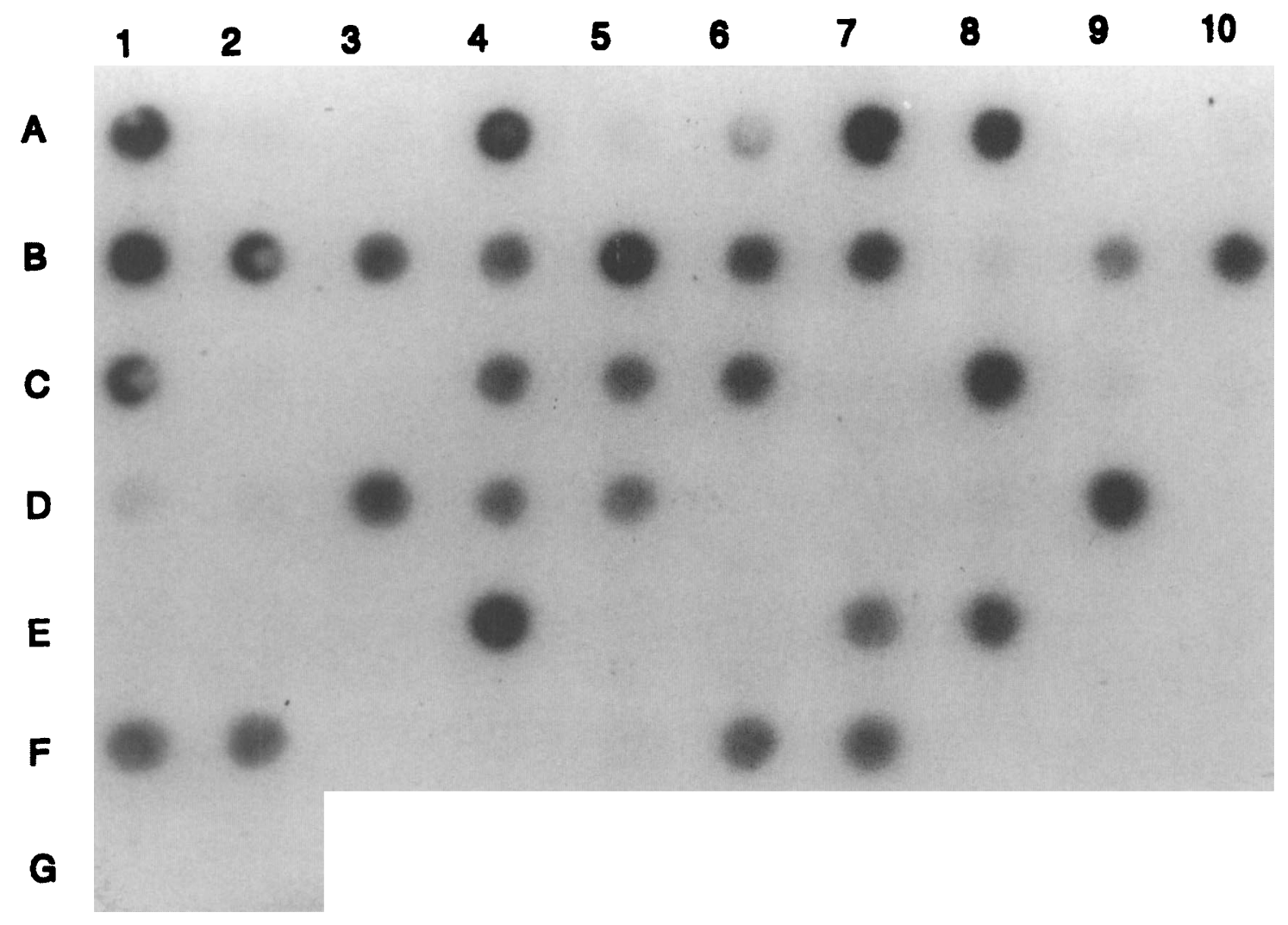

Fig. 2. Dot-blot analysis. Amplified samples were blotted on to nylon membrane and hybridised to a radioactive labelled probe specific for $M$. avium. Samples in grid numbers A1-G2 correspond to clinical isolates and reference ATCC cultures in the same order as listed in Table 1.

A

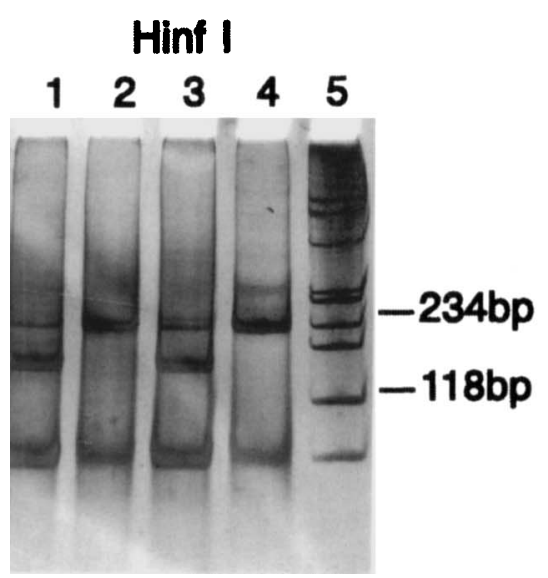

B

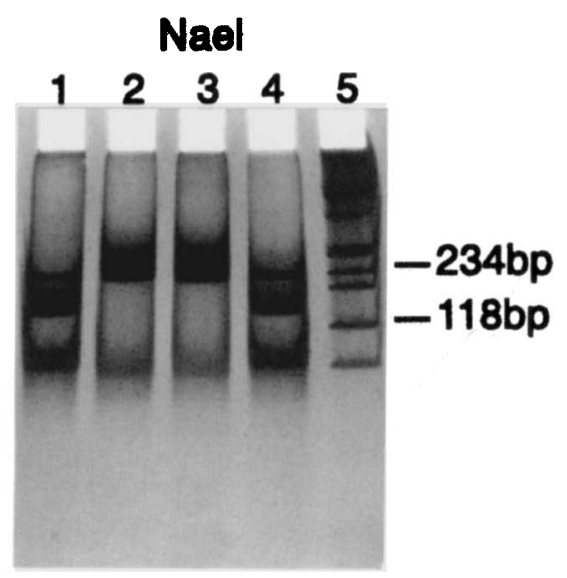

Fig 3. RFLP analysis. Ten $\mu$ l of amplified PCR product from isolates 7 (lane 1), 9 (2), 10 (3) and 39 (4) were digested with $\mathrm{Hin} \mathrm{fI}(\mathbf{A})$ and $\mathrm{Nae}$ I (B) and the restricted products were detected by gel electrophoresis. Lane 5 contains PhiX/ Hae III as marker in both figures.

(ATCC 15679 and 35717 ), only six (14.3\%) of the 42 clinical isolates could be identified as $M$. avium by the previously described RFLP method (Table 1). The same six isolates were also probe positive (Fig. 2). A sample result is shown in Fig. 3. The two clinical isolates identified as $M$. gordonae, (nos. 6 and 26) were also probe positive for $M$. avium sequences by this technique. Furthermore, isolate 6 gave an RFLP pattern that was indistinguishable from those of some $M$. avium isolates.

\section{Sequence analysis}

Direct sequence analysis of amplified dnaJ products confirmed the results obtained with the $M$. aviumspecific probe and RFLP analysis with some isolates. 

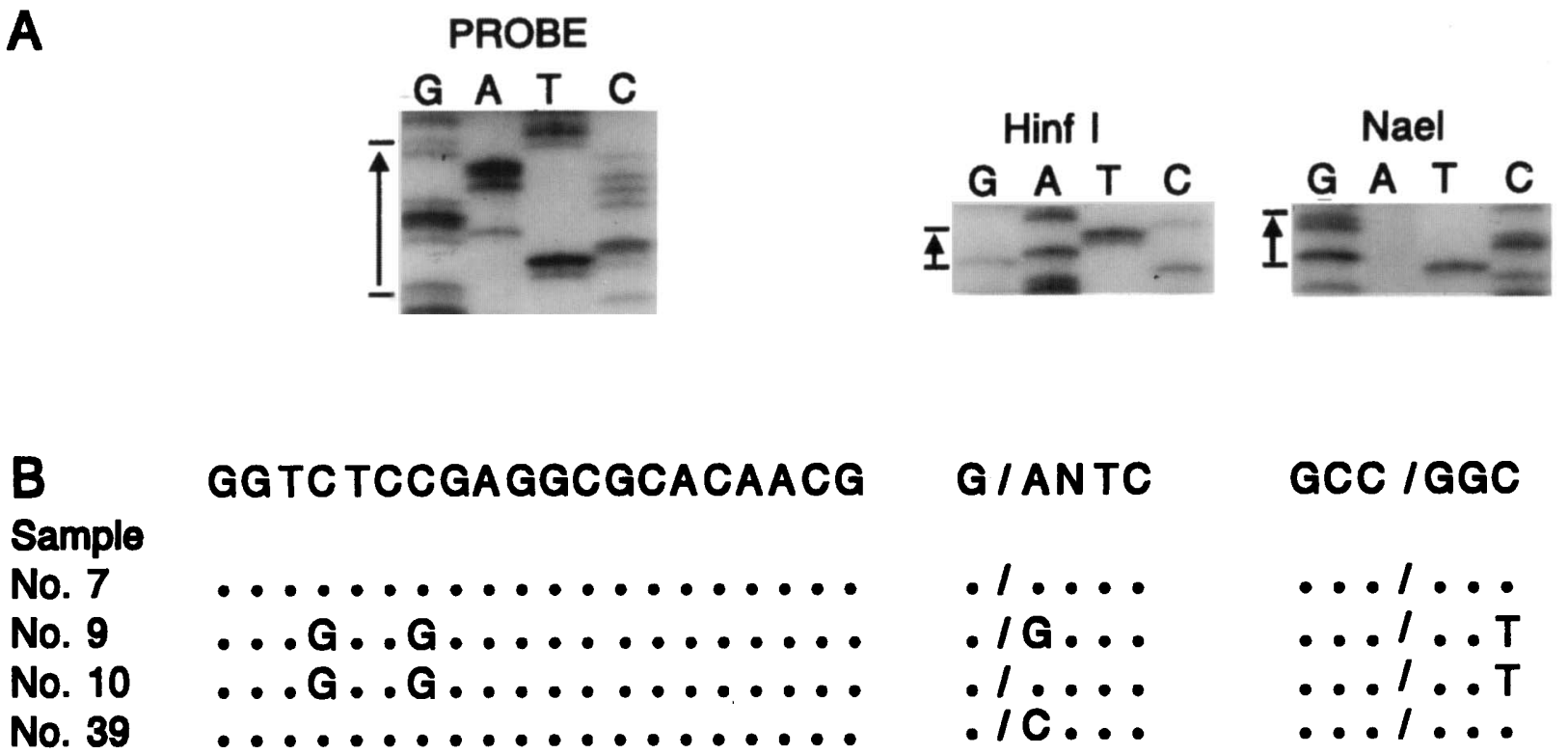

G / ANTC

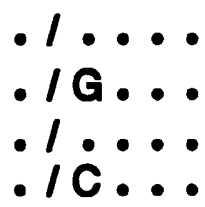

GCC /GGC

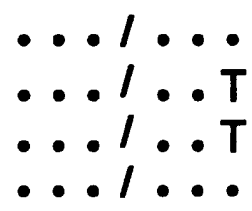

Fig 4. Direct sequence analysis of PCR products. Direct sequence analysis of amplified dnaJ gene products was done for $M$. avium isolates 7, 9, 10 and 39. A, sequence information for the probe and Hin $\mathrm{fl}$ and NaeI recognition regions for sample 7, B, sequences for samples 7, 9, 10 and 39 were compared to published sequences for the probe [7], Hin $\mathrm{fI}$ and NaeI recognition regions and a summary of the results is shown.

For example, sequence analysis (Fig. 4) of isolate 7, which was probe positive, gave the expected probebinding and restriction endonuclease cutting sequence, unlike isolate 9. This finding suggests that isolate 9 may not be $M$. avium, although initially identified as such by biochemical tests. Sequence information also confirmed the RFLP pattern and probe result obtained for $M$. avium isolate 10. A further example of variation is that seen in isolate 39 , which hybridised strongly to the probe, but did not give the characteristic RFLP pattern. This result, showing loss of the restriction site, was confirmed by sequence analysis (Fig. 4). Although samples from all the isolates were not sequenced, it is interesting to note that $20(76.9 \%)$ of the $26 \mathrm{M}$. avium clinical isolates that hybridised to the probe did not give the characteristic RFLP pattern.

\section{Discussion}

The aim of this study was to use the SSCP technique to facilitate the identification and classification of amplified $d n a J$ gene sequences for species identification of mycobacteria. The finding that clinical isolates of $M$. tuberculosis with different fingerprint patterns all gave the same distinct SSCP banding patterns, which were different from those of atypical mycobacteria, is potentially useful as it indicates that the dnaJ gene is highly conserved in this species. This methodology provides a relatively simple and quick laboratory procedure for distinguishing $M$. tuberculosis from other clinical important mycobacteria, such as $M$. avium.
It has been suggested that PCR DNA amplification of the dnaJ gene of mycobacteria and subsequent speciesspecific oligonucleotide hybridisation or RFLP analysis of PCR products are useful for differential diagnosis of tuberculosis and non-tuberculous mycobacterial infections $[7,8]$. However, only a limited number of clinical isolates has been analysed thus far. Although the aim of this study was to use the SSCP technique to facilitate the identification of Mycobacterium species, the most important finding was the considerable strainspecific dnaJ gene variation observed amongst atypical mycobacteria. The clinical isolates used in this study consisted mainly of $M$. avium and only $62 \%$ of these hybridised with the $M$. avium-specific probe. However, only six (14\%) of these 42 isolates could be identified as $M$. avium by both probe and RFLP analysis. It is possible that some of the isolates may have been incorrectly identified by the biochemical testing, but it is unlikely that the tests would be wrong for $86 \%$ of the isolates. Independent Genprobe of HPLC analysis could help to define the nature of these isolates, although this was not done in the present study. Of the $M$. avium isolates that hybridised to the probe, $77 \%$ gave negative results with one or both of the two endonucleases used for RFLP typing. This finding was supported by sequence analysis. Although the probe and RFLP analysis correctly identified the two reference strains of $M$. avium, the results suggest considerable strain-specific variation in the dnaJ gene of mycobacteria other than $M$. tuberculosis in a large scale analysis of clinical isolates. This finding is also supported by the variation observed after SSCP analysis of the amplified region of the dnaJ gene. However, it must be noted that nucleotide variations in regions 
outside the probe and restriction endonuclease recognition sites may also have an influence on the SSCP result. Thus, this SSCP technique may be valuable for discriminating between tuberculosis and non-tuberculous mycobacterial infection, but may not be suitable for species identification of atypical mycobacteria.

The SSCP pattern of the two clinical isolates of $M$. scrofulaceum not only differed from each other, but were also different from the ATCC 19981 control included in this study. A similar result was obtained with a number of clinical isolates of $M$. gordonae. Two of these latter isolates also hybridised with the $M$. avium-specific probe but one did not give the characteristic RFLP pattern for $M$. avium. This crosshybridisation between strains again suggests some form of genetic drift in the dnaJ gene. The lower mol.wt product detected for two of the $M$. gordonae isolates (c. $230 \mathrm{bp}$ instead of $236 \mathrm{bp}$ ) was equally unexpected for this primer set and increased numbers of isolates and further sequence data are necessary to study dnaJ in this species.

Members of the $M$. avium complex are the commonest non-tuberculous mycobacteria associated with human disease [1]. Various subtyping techniques have been described for these species, including seroagglutination, thin layer chromatography, plasmid analysis, gas chromatography-mass spectrometry, probes and RFLPs $[3,7,9,18,19]$. Serotyping has been used most frequently, but is limited by the high proportion of isolates that cannot be typed [20] and results are not consistent between laboratories [4]. Furthermore, there may be as many as 28 serovars [1] and the prevalence and serovar distribution vary in different geographical areas [21]. The results of serotyping do not correlate well with genotyping methods [22]. The homogeneity observed in this study in dnaJ sequences of different strains of $M$. tuberculosis compared to the heterogeneity of this amplified region in different strains of $M$. avium is significant. It has long been recognized that the $M$. avium complex is genetically diverse but, despite this diversity, the $M$. avium complex does form a welldefined phylogenetic group that is distinct from the phenotypically similar $M$. scrofulaceum, $M$. xenopi and $M$. celatum [23]. This is supported by the results of this study, as no band-sharing was seen in SSCP between $M$. avium and $M$. scrofulaceum.

The considerable strain-specific dnaJ gene variation reported in this study, even in the so-called conserved sites for M. avium (probe and restriction sites), may support the above findings and this technology or gene sequence may not be a good method for strain identification by PCR amplification, as suggested elsewhere $[7,8]$. The results also emphasise the importance of testing a large collection of clinical isolates and suggest that examination of only a few reference cultures may give biased results. The variation also suggests that the development of a species-specific PCR-based test for this gene is not to be recommended.

The authors gratefully acknowledge the Glaxo TB initiative and Tygerberg Hospital for financial assistance and R. Warren, I. Wiid, C. Werely, Madelene Richardson and F. Cilliers for DNA fingerprinting data.

\section{References}

1. Wolinsky E. Mycobacterial diseases other than tuberculosis. Clin Infect Dis 1992; 15: 1-12.

2. Horsburgh CR, Cohn DL, Roberts RB et al. Mycobacterium avium-M. intracellulare isolates from patients with or without acquired immunodeficiency syndrome. Antimicrob Agents Chemother 1986; 30: 955-957.

3. Kiehn TE, Edwards FF, Brannon $P$ et al. Infections caused by Mycobacterium avium complex in immunocompromised patients: diagnosis by blood culture and fecal examination, antimicrobial susceptibility tests and morphological and seroagglutination characteristics. J Clin Microbiol 1985; 21: 168173.

4. Wayne LG, Good RC, Tsang A et al. Serovar determination and molecular taxonomic correlation in Mycobacterium avium, Mycobacterium intracellulare, and Mycobacterium scrofulaceum: a cooperative study of the International Working Group on Mycobacterial Taxonomy. Int $J$ Syst Bacteriol 1993; 43: 482489.

5. Yakrus MA, Good RC. Geographic distribution, frequency, and specimen source of Mycobacterium avium complex serotypes isolated from patients with acquired immunodeficiency syndrome. J Clin Microbiol 1990; 28: 926-929.

6. Cook SM, Bartos RE, Pierson CL, Frank TS. Detection and characterization of atypical mycobacteria by the polymerase chain reaction. Diagn Mol Pathol 1994; 3: 53-58.

7. Takewaki S-I, Okuzumi K, Ishiko H, Nakahara K-I, Ohkubo A, Nagai R. Genus-specific polymerase chain reaction for the mycobacterial dnaJ gene and species-specific oligonucleotide probes. J Clin Microbiol 1993; 31: 446-450.

8. Takewaki S-I, Okuzumi K, Manabe I et al. Nucleotide sequence comparison of the mycobacterial dnaJ gene and PCR-restriction fragment length polymorphism analysis for identification of mycobacterial species. Int $J$ Syst Bacteriol 1994; 44: 159-166.

9. Telenti A, Marchesi F, Balz M, Bally F, Aöttger EC, Bodmer T. Rapid identification of mycobacteria to the species level by polymerase chain reaction and restriction enzyme analysis. $J$ Clin Microbiol 1993; 31: 175-178.

10. Vaneechoutte M, de Beenhouwer H, Claeys G et al. Identification of Mycobacterium species by using amplified ribosomal DNA restriction analysis. J Clin Microbiol 1993; 31: 20612065.

11. Horsburgh CR. Mycobacterium avium complex infection in the acquired immunodeficiency syndrome. N Engl J Med 1991; 324: 1332-1338.

12. Orita $M$, Iwahana $H$, Kanazawa $H$, Hyashi $K$, Sekiya $T$. Detection of polymorphisms of human DNA by gel electrophoresis as single-strand conformation polymorphisms. Proc Natl Acad Sci USA 1989; 86: 2766-2770.

13. Heym B, Honoré N, Truffot-Pernot $\mathrm{C}$ et al. Implications of multidrug resistance for the future of short-course chemotherapy of tuberculosis: a molecular study. Lancet 1994; 344: 293-298

14. Fisher S. Western Cape Regional Services Council: Annual report of the Department of Health Services, South Africa. 1990: 35-51.

15. Roberts GD, Koneman EW, Kim YK. Mycobacterium. In Balows A, Hausler WJ, Herrmann KL, Isenberg HD, Shadomy HJ (eds) Manual of clinical microbiology, 5th edn. Washington, DC, American Society for Microbiology. 1991: 304-339.

16. Wiid IJF, Werely C, Beyers N, Donald P, van Helden PD. Oligonucleotide (GTG) $s$ as a marker for Mycobacterium tuberculosis strain identification. J Clin Microbiol 1994; 32: 1318-1321.

17. van Soolingen D, de Haas PEW, Hermans PWM, Groenen PMA, van Embden JDA. Comparison of various repetitive DNA 
elements as genetic markers for strain differentiation and epidemiology of Mycobacterium tuberculosis. J Clin Microbiol 1993; 31: 1987-1995.

18. Hellyer TJ, Brown IN, Dale JW, Easmon CSF. Plasmid analysis of Mycobacterium avium-intracellulare (MAI) isolated in the United Kingdom from patients with and without AIDS. $J$ Med Microbiol 1991; 34: 225-231.

19. Tsang AY, Denner JC, Brennan PJ, McClatchy JK. Clinical and epidemiological importance of typing of Mycobacterium avium complex isolates. J Clin Microbiol 1992; 30: 479-484.

20. Dawson DJ. Infection with Mycobacterium avium complex in
Australian patients with AIDS. Med J Aust 1990; 153: 466-468.

21. Falkinham JO, Parker BC, Gruft H. Epidemiology of infection by nontuberculous mycobacteria. I. Geographic distribution in the eastern United States. Am Rev Respir Dis 1980; 121: 931937.

22. Frothingham R, Wilson $\mathrm{KH}$. Sequence-based differentiation of strains in the Mycobacterium avium complex. $J$ Bacteriol 1993; 175: $2818-2825$.

23. Frothingham R, Wilson KH. Molecular phylogeny of the Mycobacterium avium complex demonstrates clinically meaningful divisions. $J$ Infect Dis 1994; 169: 305-312. 